\title{
Double Bundle Posterior Cruciate Ligament Reconstruction in 100 Patients at a Mean 3 Years Follow up: Outcomes were Comparable to an Anterior Cruciate Ligament Reconstructions
}

\author{
Jorge Chahla, MD, PhD¹, Mark Cinque ${ }^{2}$, Andrew G. Geeslin, MD³ , Grant Dornan², Gilbert Moatshe, MD², Robert F. \\ LaPrade, MD, $\mathrm{PhD}^{4}$
}

${ }^{1}$ Santa Monica Orthopaedic \& Sports Medicine Group Program, Santa Monica, CA, USA, ${ }^{2}$ Steadman Philippon Research Institute, Vail, CO, USA, ${ }^{3}$ Steadman Philippon Research Institute, Kalamazoo, MI, USA, ${ }^{4}$ The Steadman Clinic, Vail, CO, USA.

Objectives: 1) To report on the outcomes after double-bundle PCL reconstructions in isolated versus combined injuries and acute versus chronic PCL tears and 2) to compare the outcomes of isolated double-bundle PCL reconstruction (DB PCLR) to isolated $A C L$ reconstruction (ACLR).

Methods: All patients who underwent a primary arthroscopic assisted DB PCLR for grade-III isolated or combined PCL injuries between May 2010 and March 2015 were reviewed. Patient reported outcome scores (Lysholm, Tegner, Western Ontario and McMaster Universities Arthritis Index (WOMAC), 12 item Short Form Health Survey (SF-12) Physical Component Summary (PCS) and patient satisfaction with outcome) and objective posterior stress radiographs were collected preoperatively and at a minimum of two years postoperatively. Cohort subanalyses comparing isolated versus combined, and acute versus chronic PCL reconstructions were also performed. Patients who underwent isolated ACLR over the same inclusion period were selected as a comparison group.

Results: One hundred patients that underwent DB PCLR were included in this study. There were 31 isolated PCL injuries and 69 combined PCL injuries and the mean follow-up was 2.9 years (range 2-6 years). The median Tegner activity score improved from 2 to 5 , Lysholm from 48 to 86 , WOMAC from 35.5 to 5 , and SF-12 PCS from 34 to 54.8 (all $p$ values $<0.001$ ). The mean side-to-side difference $(S S D)$ in posterior tibial translation on kneeling stress radiographs improved from $11.0 \mathrm{~mm}$ preoperatively to $1.6 \mathrm{~mm}$ postoperatively $(p<0.001)$. There were no significant differences in postoperative functional scores between isolated PCL reconstructions and combined PCL reconstructions (all $p$ values $>0.229$ ). The mean SSD in postoperative posterior tibial translation on stress radiographs was $1.2 \pm 1.1 \mathrm{~mm}$ for isolated $\mathrm{PCL}$ tears and $1.7 \pm 2.2 \mathrm{~mm}$ for combined PCL tears. The improvement in posterior tibial translation from preoperative to postoperative was significant for both the isolated and combined PCL injury groups $(p<0.001)$. Only the Tegner score $(p<0.001)$ and patient satisfaction $(p=0.011)$ were significantly different postoperatively between acute and chronic reconstructions, both favoring acutely treated PCL injuries. The mean SSD in posterior tibial translation on stress radiographs improved from $11.6 \pm 3.1 \mathrm{~mm}$ preoperatively to $1.9 \pm$ $2.5 \mathrm{~mm}$ postoperatively $(p<0.001)$ for acute $P C L$ tears, and $10.3 \pm 3.7 \mathrm{~mm}$ to $1.2 \pm 1.0 \mathrm{~mm}(p<0.001)$ for chronic $P C L$ tears. There were no significant differences in postoperative outcome scores between patients that underwent an isolated ACLR or isolated DB PCLR [all $p$ values $>0.064$ ].

Conclusion: Significantly improved functional and objective outcomes were observed after anatomic-based DB PCLR at a mean 3 years follow-up, regardless of concomitant ligamentous pathology or timing to surgery. Posterior tibial translation was restored to near normal after DB PCLR. Additionally, contrary to previous reports, similar results were achieved compared to a control isolated ACLR cohort.

\footnotetext{
This open-access article is published and distributed under the Creative Commons Attribution - NonCommercial - No Derivatives License (http://creativecommons.org/licenses/by-nc-nd/4.0/), which permits the noncommercial use, distribution, and reproduction of the article in any medium, provided the original author and source are credited. You may not alter, transform, or build upon this article without the permission of the Author(s). For article reuse guidelines, please visit SAGE's website at http://www.sagepub.com/journals-permissions.
} 
Table 1: Patient demographics and preoperative outcome scores demonstrating that the initial status of both cohorts was comparable. Data presented as counts, mean - SD or median [1st quartile, 3rd quartile], unless otherwise noted. N/A=Not applicable; FET= Fisher's exact tests; $(x 2)=$ chi-squared tests; MWU=Mann-Whitney U-tests

\begin{tabular}{|c|c|}
\hline Variable & $\begin{array}{l}\text { PCL Reconstruction Cohort } \\
(n=100)\end{array}$ \\
\hline Patients & 100 \\
\hline Age & Mean 31.7 (range, 14-66) \\
\hline Gender & $\begin{array}{l}\text { Male: } 77 \\
\text { Female: } 23\end{array}$ \\
\hline Follow-Up Interval (years) & Mean 2.9 (range, 2-6) \\
\hline Chronicity & Acute: 52 Chronic: 48 \\
\hline Meniscus Tear Distribution & $\begin{array}{l}\text { None: } 54 \text { Medial Meniscus: } 23 \\
\text { Lateral Meniscus: } 16 \text { Medial } \& \\
\text { Lateral Meniscus: } 7\end{array}$ \\
\hline $\begin{array}{l}\text { Outerbridge Grade IV } \\
\text { Chondral lesions (Grade, } \\
\text { Location) }\end{array}$ & Full thickness lesions: 11 \\
\hline $\begin{array}{l}\text { Preoperative Outcome } \\
\text { Scores }\end{array}$ & $\begin{array}{l}\text { PCL Reconstruction Cohort } \\
(n=100)\end{array}$ \\
\hline Tegner Activity Scale & $2[1,3]$ \\
\hline Lysholm Score & $49.6 \pm 25.1$ \\
\hline $\begin{array}{l}\text { Western Ontario and } \\
\text { McMaster Universities } \\
\text { Arthritis Index Total }\end{array}$ & $38.7 \pm 27.9$ \\
\hline $\begin{array}{l}\text { Short Form-12 Physical } \\
\text { Health Composite Score }\end{array}$ & $37.6 \pm 10.9$ \\
\hline
\end{tabular}

ACL Reconstruction Cohort $(n=141)$

141

Mean 35.2 (range, 14-81)

Male: 63

Female: 78

Mean 3.1 (range, 2-7)

Acute: 93 Chronic: 48

None: 66 Medial Meniscus: 39 Lateral Meniscus: 21 Medial \& Lateral: Meniscus: 15

None

ACL Reconstruction Cohort ( $n=141)$

$2[1,5]$

$51.0 \pm 23.2$

$35.2 \pm 23.1$

$40.3 \pm 9.7$
N/A

P-Value

$\mathrm{N} / \mathrm{A}$

$0.042^{*}$

(MWU)

$<0.001^{*}$

(FET)

0.289

(MWU)

0.033

(FET)

$0.590\left(x^{2}\right)$

P-Value

0.135

(MWU)

0.691 (ttest)

$0.333(\mathrm{t}-$ test)

$0.015^{*}$ (ttest) 


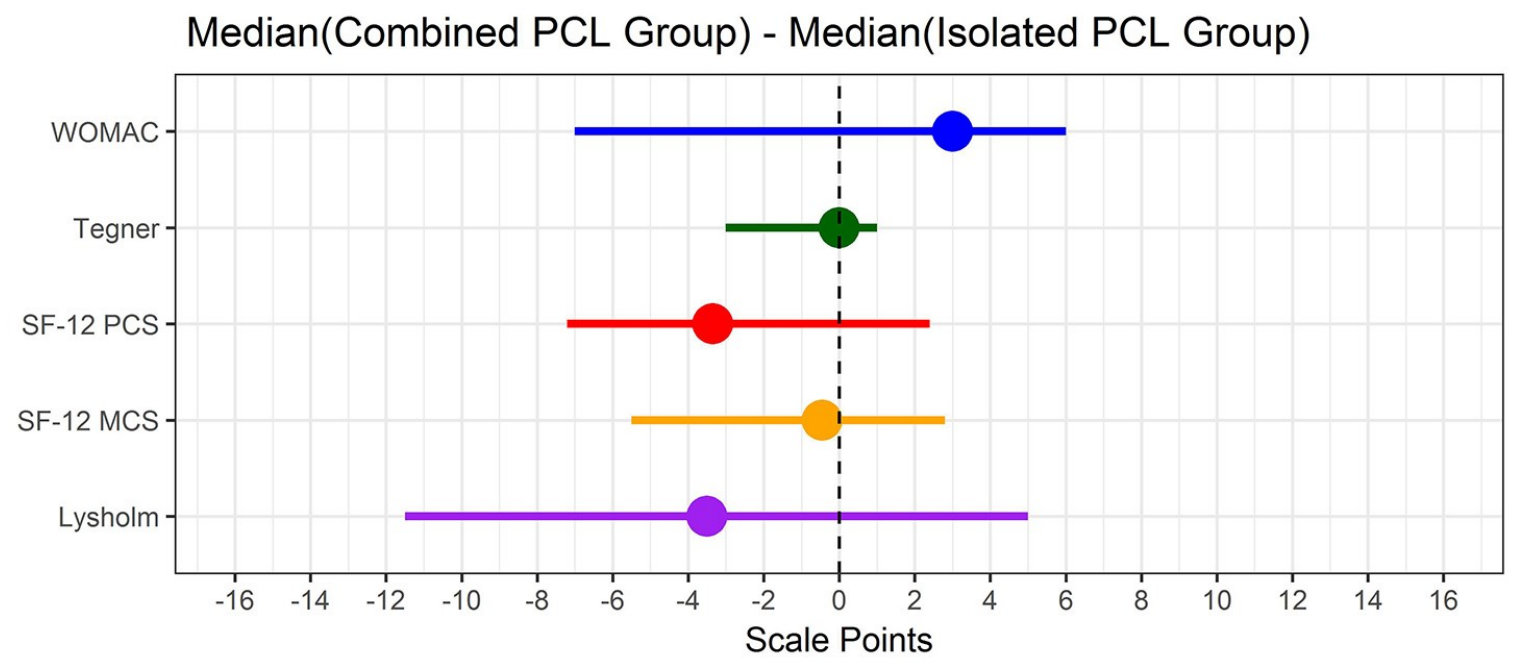

Median(Chronic PCL Group) - Median(Acute PCL Group)

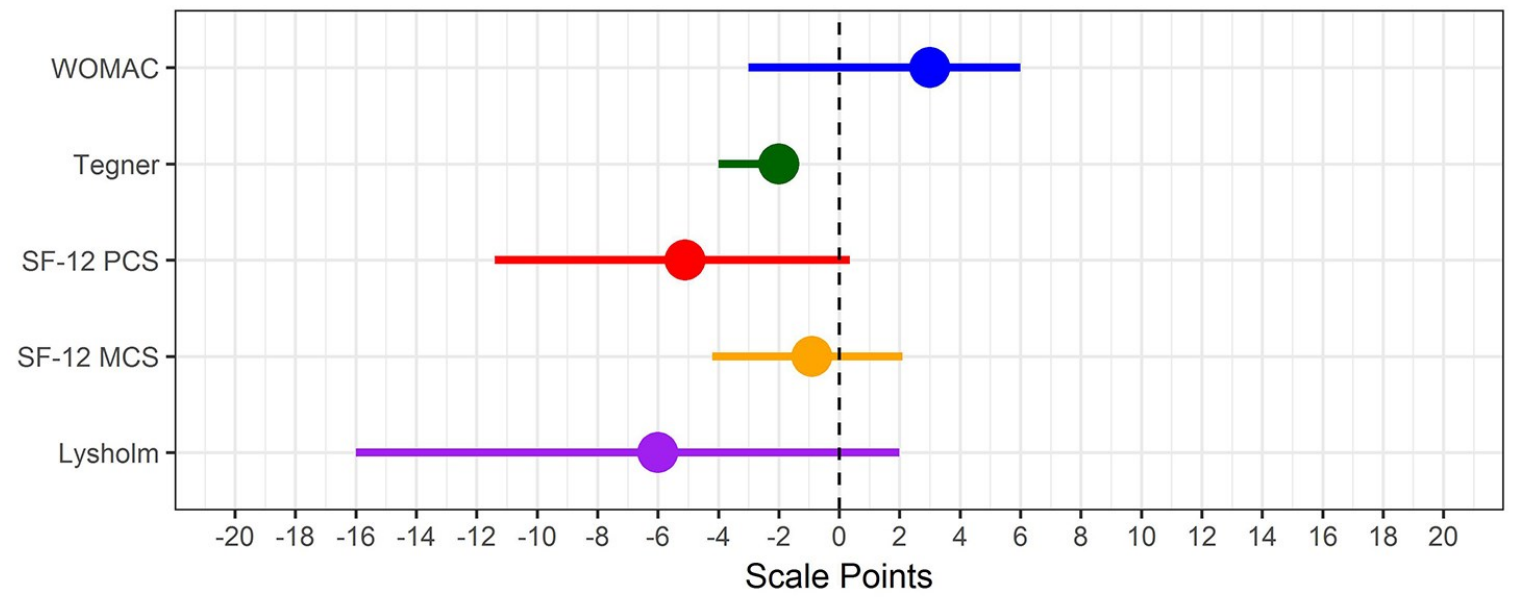

Median(Isolated PCL Group) - Median(Isolated ACL Group)

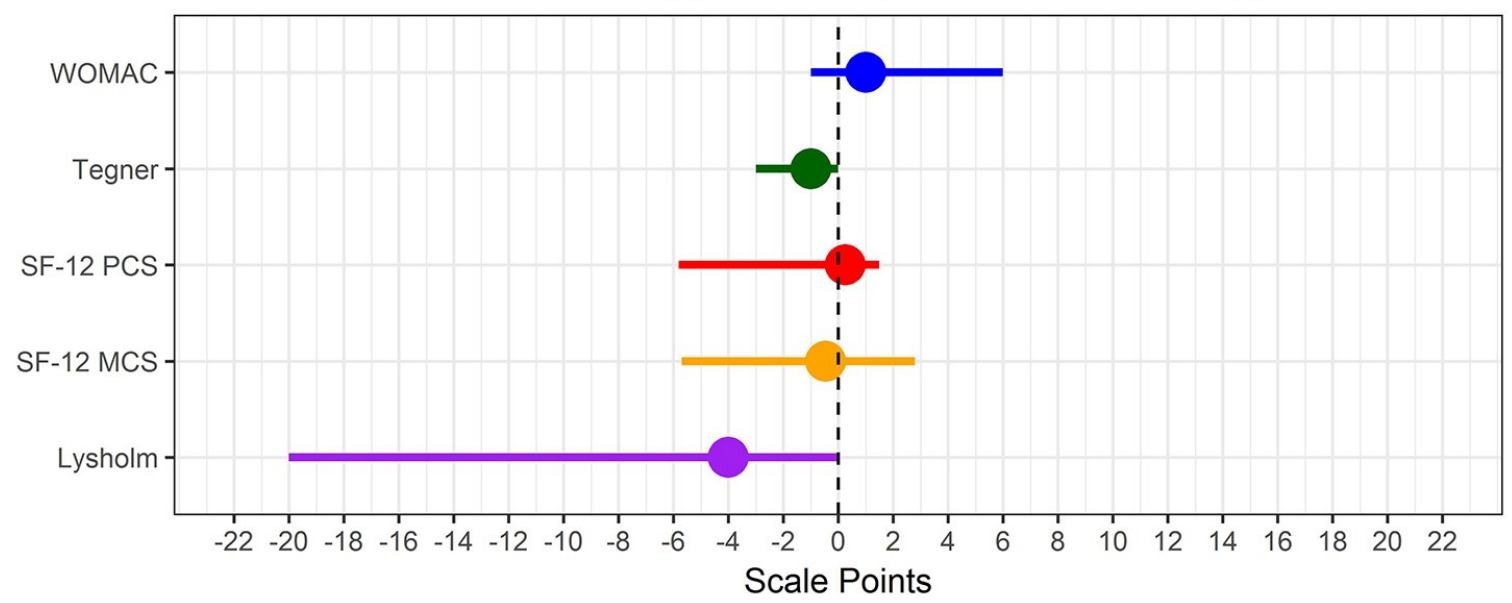

Fig 1. Difference in medians for functional outcome scales. Horizontal lines indicate $95 \%$ bootstrap confidence intervals.

The Orthopaedic Journal of Sports Medicine, 6(7)(suppl 4)

DOI: $10.1177 / 2325967118 S 00062$

(CThe Author(s) 2018 